\title{
Progress of Lymph-Targeted Contrast Agents and Their Application in Diagnosis of Tumor Lymphatic Metastasis
}

\author{
Ying Zhang, ${ }^{\dagger, a, b}$ Jie Wang, ${ }^{\dagger, a}$ Jiejun Cheng, ${ }^{\dagger, c}$ Yu Qiao, ${ }^{a, b}$ Gang Huang, ${ }^{*, d}$ \\ Jun Zhu, ${ }^{*, b}$ and Dannong $\mathrm{He}^{*, a, b}$ \\ ${ }^{a}$ College of Materials Science and Engineering, Shanghai Jiao Tong University, Shanghai 200240, China \\ ${ }^{b}$ National Engineering Research Center for Nanotechnology, Shanghai 200241, China \\ ${ }^{\mathrm{c}}$ Department of Radiology, Shanghai Renji Hospital, Shanghai Jiao Tong University School of Medicine, \\ Shanghai 200127, China \\ ${ }^{d}$ Shanghai University of Medicine \& Health Sciences, Shanghai 200241, China
}

\begin{abstract}
Lymph node metastasis is an important aspect for tumor metastasis, which accounts for a majority of cancer death. Non-invasive imaging techniques play a more important role than traditional lymph node biopsy for diagnosis of metastatic lymph nodes. However, non-specific information of lymph node such as size and morphology could only be obtained in traditional imaging techniques, which always bring about mission detection. Thus, lymph-targeted contrast agents are attracting much interest. Presently, most contrast agents are based on the mechanism of passive targeting, which could be affected by the number and function of macrophages in the lymph nodes. Therefore, it is beneficial to study the tumor lymphatic metastases through developing new lymph-targeted contrast agents, which could bring fresh prospect for tumor diagnosis and treatment. The paper reviews the progress of lymph-targeted contrast agents and their diagnosis technology for cancer.
\end{abstract}

Keywords lymph-targeted contrast agents, tumor lymphatic metastasis, diagnosis techniques

\section{Introduction}

Tumor metastasis is an important cause of death in patients with cancer. More than $80 \%$ of the patients died of tumor recurrence and metastasis. ${ }^{[1]}$ Early detection, diagnosis and treatment of cancer can help to prevent deterioration of tumor and decrease recurrence and metastasis of tumor. ${ }^{[2]}$ Therefore, it is of great significance to develop new methods for early diagnosis and treatment of cancer.

Generally, the modes of tumor metastasis include hematogenous, implantation and lymphatic metastasis. ${ }^{[3]}$ Hematogenous $^{[4]}$ and implantation metastasis ${ }^{[5]}$ can be examined by detecting the number of tumor cells in blood and abdominal cavity, ${ }^{[6]}$ realizing early noninvasive diagnosis of tumor metastasis. ${ }^{[7]}$ Furthermore, clinical imaging examination of lymph nodes has achieved non-invasive diagnosis of lymphatic metastasis. ${ }^{[8]}$ The criterion of judgment lies on non-specific information of lymph nodes, ${ }^{[9]}$ such as morphology, size,${ }^{[10]}$ fusion and necrosis, ${ }^{[11]}$ which may lead to possible false positive and false negative. For example, some normal sized lymph nodes may have occurred in tumor invasion and metastasis. ${ }^{[12,13]}$ Therefore, it is very important to develop diagnosis technologies of lymph node for warning lymphatic metastasis of tumor. Lymph-targeted contrast agents are attracting much interest. ${ }^{[14]}$ Current clinical imaging technologies include magnetic resonance imaging (MRI), contrast-enhanced ultrasound computed tomography (CT), and so on. ${ }^{[15]}$ Presently, most contrast agents are based on the mechanism of passive targeting, which could be affected by the number and function of macrophages in the lymph nodes. ${ }^{[16]}$

It was found that the functions and internal structures of lymph nodes were significantly affected by invasion of tumor cells. ${ }^{[17]}$ Some unique features of contrast agents using pathological lesion site can selectively target tumor tissue, but their efficiencies are very low as a passive target. ${ }^{[18]}$ Only a small part of contrast agents can reach tumor sites by enhanced permeability and retention (EPR) effect; however, most drugs still circulates in the blood. Therefore, imaging diagnosis technology needs to rely on targeted functional groups to achieve active targets. ${ }^{[19]}$ A targeted contrast agent is connected to an imaging agent by a target molecule with a specific function such as antibody or ligand. ${ }^{[20]}$ Therefore, the construction of lymph node targeted contrast agents has become the direction of developing lymph node imaging technology, and it is also a hot issue in the field of biomedical engineering.

The paper reviews the progress of lymph-targeted contrast agents and their diagnosis technology for cancer.

\section{Tumor lymphatic metastasis}

Lymphatic metastasis $^{[21]}$ is an important pattern for cancer spread, ${ }^{[22]}$ which means that exfoliated tumor cells pass through lymphatic vessels, collected to a lymph node by the lymph and grown to a tumor. ${ }^{[23]}$ Furthermore, recent researches indicate that the binding of vascular endothelial growth factor (VEGF) ${ }^{[24]}$ from tumor cells and their receptor ${ }^{[25]}$ on lymphatic endothelial cells can induce the formation of lymphatic tube inside or outside of tumor tissue ${ }^{[26]}$ and within sentinel lymph node (SLN), which promotes tumor metastasis to the lymphatic system. When tumor cells arrive at the SLN, new lymph-vessels appear greatly in lymph nodes (Figure 1). Furthermore, they can also arrive at the next lymph node to reduce new lymph-vessel, which would produce secondary or distal lymphatic metastasis. ${ }^{[27]}$

* E-mail: hdn_nercn@163.com

Received October 2, 2016; accepted November 30, 2016.

${ }^{\dagger}$ These authors contributed equally to this work. 


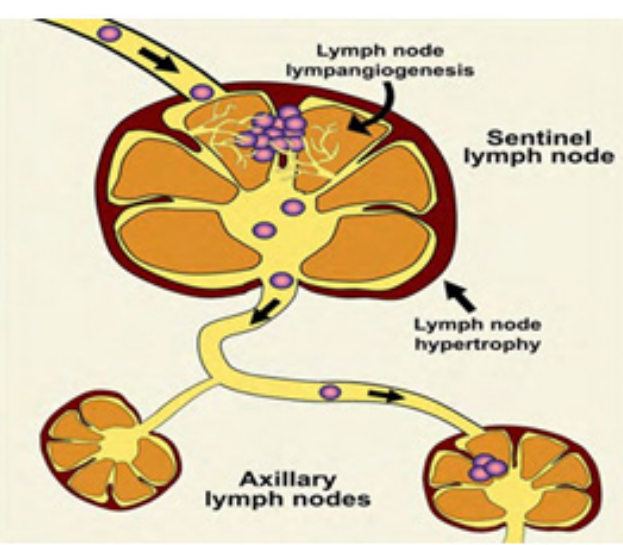

Figure 1 Transfer of tumor cells in sentinel lymph node.

Many researchers found that lymphatic metastasis is popular in lung, kidney, breast, melanoma, and other epithelial cancer. Some achievements were obtained. For example, Chuck et al. ${ }^{[28]}$ observed tumor metastasis in mice with breast cancer. Figure 2 shows a mice breast injected with fluorescent tumor cells after 6 and $12 \mathrm{w}$. It can be seen that the tumor cell has been transferred to SLN $12 \mathrm{w}$ later.

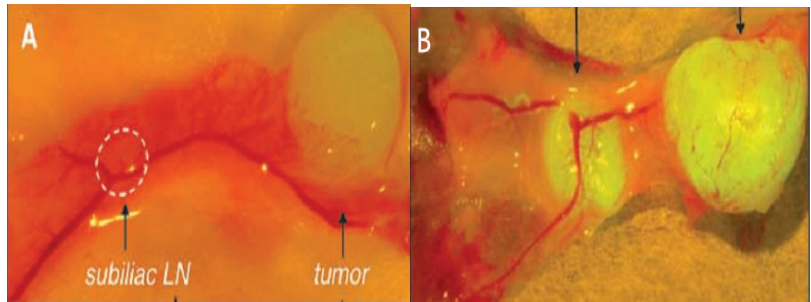

Figure 2 Imaging of mice breast containing fluorescent tumor cell after $6(\mathrm{~A})$ and $12 \mathrm{w}(\mathrm{B})$.

It is critical for the diagnosis of lymph nodes to judge lymphatic metastasis. Initially, the lymph node biopsy is considered to be an important indicator for assessing tumor lymphatic metastasis. However, some drawbacks such as high cost and invasive will bring a lot of complications. Furthermore, some lymph nodes may be omitted outside of surgical field, resulting in the false negative of secondary or distal lymph nodes. Recently, some new technologies play an increasingly important role during the assessment of tumor lymphatic metastasis. For example, imaging technology is a non-invasive method for evaluating whole lymph nodes, which is mainly based on the diagnosis of lymph nodes invaded by tumor cells. ${ }^{[29-31]}$

\section{Diagnosis based on tumor microenvironment}

Tumor microenvironment is the internal environment of the tumor in the process of its occurrence and development, which is composed of tumor cells themselves, stromal cells, micro vascular, lymphatic capillaries, tissue fluid, numerous cytokines, a small number of infiltrating cells, and so on. ${ }^{[32]}$ Generally, normal cells stay in a relatively stable internal environment, where proliferation, differentiation, apoptosis, secretion and expression of related factors are carried out according to normal procedures. However, the procedures could be broken by the formation and development of tumor. ${ }^{[33]}$ Local nutrient conditions is unable to meet the requirements of their growth with the development of a tumor, which leads to the metastasis of their cells through inducing some new lymph and blood vessel to build a new nutrition and metabolic network. The result promotes the growth and metastasis of tumor cells. Therefore, it is realizable for tumor microenvironment to diagnose the metastasis through lymph node.

The research of key lymphatic endothelial growth factors and lymphatic endothelial markers, such as vascular endothelial growth factor-C and D (VEGF-C and VEGF-D), vascular endothelial growth factor receptor 3 (VEGFR-3), lymphatic vessel endothelial hyaluronic acid receptor-1 (LYVE-1), podoplanin, prospero-related homobox protein 1 (Prox-1), improved further the diagnosis sensitivity and specificity of lymphatic metastasis from their microenvironment. Some researchers proposed that the high lymphatic vessel density around the tumor increases chances of a tumor cell dissemination. ${ }^{[34,35]}$ Furthermore, lymphatic vessels were tested using lymphatic endothelial markers, and their densities were evaluated for judgment of lymphatic metastasis. ${ }^{[36,37]}$ Human pancreatic duct cancer cells marked with LYVE-1 into nude mice were inoculated, showing positive correlation of the formation of lymphatic vessels in and out of tumor and lymphatic metastasis. ${ }^{[38-41]}$ Moreover, the mechanism of lymphatic metastasis was also proved, meaning that VEGF-C promotes the formation of lymphatic vessels in the tumor by activating its receptor VEGFR-3 on lymphatic endothelial cells and then increases lymphatic vessels. ${ }^{[42,43]}$

\section{Diagnosis based on imaging technology}

The current clinical imaging technology relies mainly on the lymph node size, shape and their relationship of blood vessels with around organ, which is always non-specifical. The results perhaps result in the false positive due to confusion of proliferation, infection, inflammation and granuloma. Thus, the normal size of metastatic lymph nodes may be missed. It is difficult for enlargement of lymph nodes to be identified the benign or malignant metastasis. Therefore, nonspecific clinical results from imaging technology can not be achieved in the early diagnosis of tumor lymphatic metastasis at present, which makes patients miss the best period of early treatment.

\section{Lymph-targeted ultrasound imaging}

The functions and internal structures of lymph nodes will change after invasion of tumor cells, which is beneficial for making accurate and timely diagnosis of lymph nodes. Lymph-targeted contrast agents are specific contrast agents that are constructed by connecting a targeted molecule specific antibody or ligand to an imaging agent in order to make the imaging technology develop from traditional morphological imaging to micro morphology, biological metabolism, gene imaging, and other aspects. Therefore, it is the research direction to develop lymph node imaging technologies for constructing lymph-targeted contrast agents.

\section{Lymph-targeted ultrasound contrast agents}

With the development of ultrasound imaging technologies, ultrasound contrast agents with targeted ability to reach special area have become a hot research topic. Several reports ${ }^{[44-46]}$ indicated that micro-bubble ultrasound contrast agents were more likely to find lymph nodes. Wisner et al. ${ }^{[44]}$ studied the application of micro-bubble ultrasound contrast agents in imaging of lymphatic system, which showed the contrast agents have a $85 \%$ enhancement effect on the imaging of lymph nodes and lymphatic vessels. The results showed that contrast agents with submicron size possessed a better imaging effect on lymph because they went easily through lymphatic systems due to their small sizes. Furthermore, targeted micro-bubble ultrasound contrast agents were reported. Niu et al. ${ }^{[47]}$ prepared a multifunctional polymer micro-foam material, which is comprised by poly lactic acid with fluorocarbon to form the structure of poly lactic acid micro-bubble. $\mathrm{Fe}_{3} \mathrm{O}_{4}$ nanoparticles and doxorubicin are encapsulated. The material can not only be used for diagnosis and treatment of lymph nodes, but also be verified to own its targering effect on lymph nodes. Figures $3 \mathrm{~A}$ and $3 \mathrm{~B}$ show that the contrast agent was about $800-900 \mathrm{~nm}$. Figure $3 \mathrm{C}$ indicates its lymph targeting effect in ultrasonic imaging. Liu et al. ${ }^{[46]}$ reported a multifunctional lymph-targeted platform based on $\mathrm{Mn} @ \mathrm{mSiO}_{2}$ nanocom- 
posites combining perfluorinated bromooctane (PFOB) for dual-mode imaging. Furthermore, HA molecule was modified to the nanocomposites, used for in vitro and in vivo targeting of the lymph system (Figure 4). The result showed that the nanocomposites can be designed with high lymph targeting efficiency and a dual-mode contrast agent for both ultrasound and MR imaging for the lymph system.

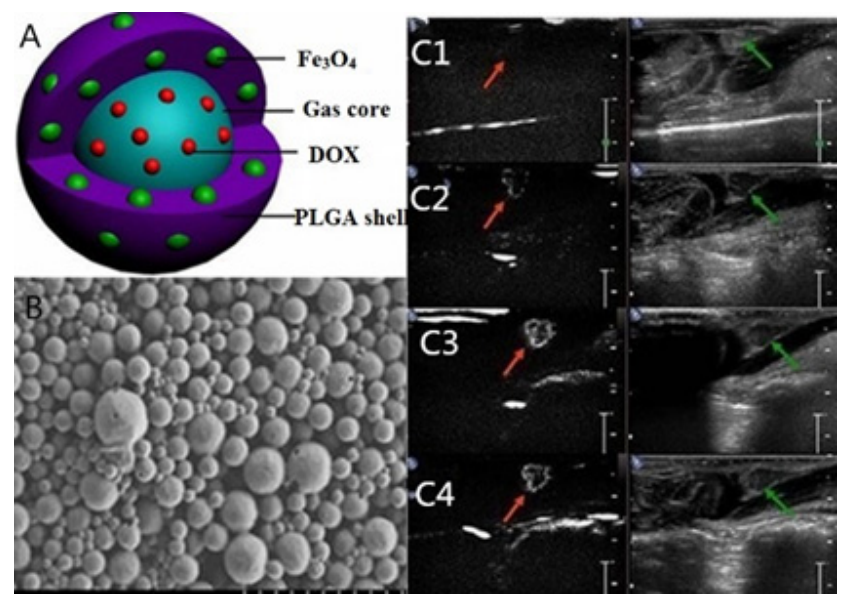

Figure 3 (A) Structure schematic diagram of micro-bubble contrast agent, (B) SEM of microbubble contras agent, and (C) ultrasonic imaging of rabbit tumor lymph node before and after contrast agent injectied.

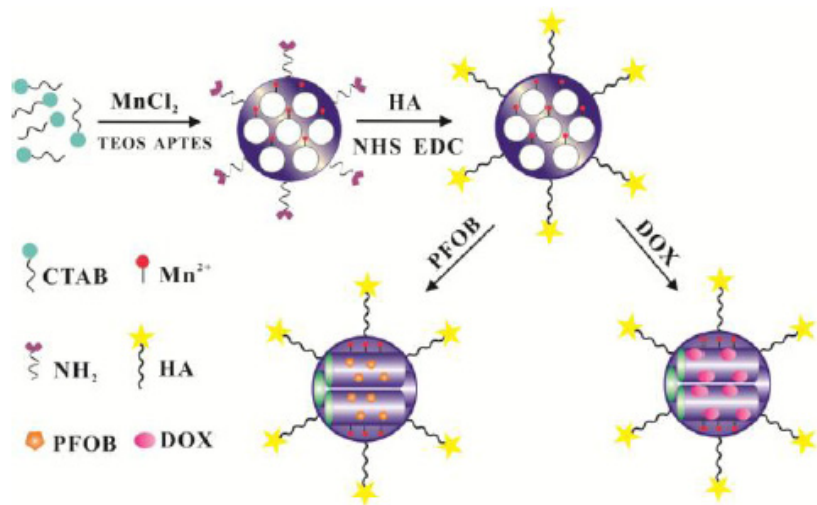

Figure 4 Schematic illustration of the formation of multifunctional lymph-targeted platform.

\section{Lymph-targeted magnetic resonance imaging}

The present research on lymph node targeted magnetic resonance imaging agents includes mainly large molecular/Gd-DTPA complex, ${ }^{[47-51]}$ protein/Gd-DTPA complex, ${ }^{[52]}$ super paramagnetic iron oxide particles (USPIO) ${ }^{[53,54]}$ and so on. The contrast agents could be based on the phagocytic mechanism and take effective agglomeration in the lymph nodes, which improves the signal to noise ratio. Thus, the differences between benign and malignant lymph nodes could be easily observed. Yan et al.$^{[5]}$ connected three different concentrations of Gd-DTPA on the hydroxyl of dextran to obtain a dextran-DTPAGd contrast agent. The contrast effect was obviously enhanced after nest site intravenous injection of dextran-DTPA-Gd in normal rabbits. The contrast effect of contrast agent on lymph nodes was gradually obvious with increase of imaging time (Figure 5). However, long imaging time is an obvious disadvantage of the contrast agent. One report ${ }^{[56]}$ indicated that a Gd-DTPA complex contrast effect obtained from Gd-DTPA marked on polyglocose-associaled marcrocomplx could be accumulated largely after injected for $24 \mathrm{~h}$.

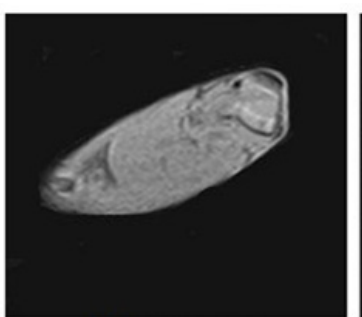

$\mathbf{B}_{1}$ before injection

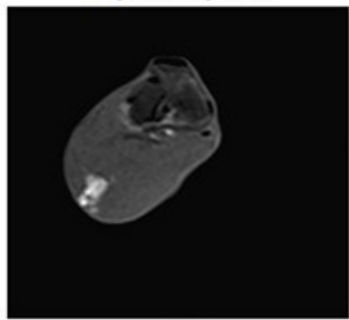

$\mathrm{B}_{3} 1 \mathrm{~h}$

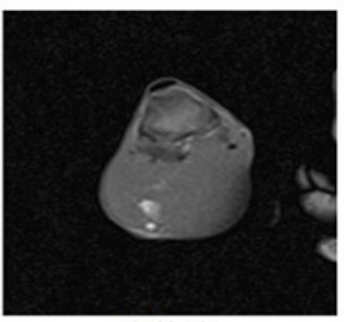

$\mathrm{B}_{2} 30 \mathrm{~min}$

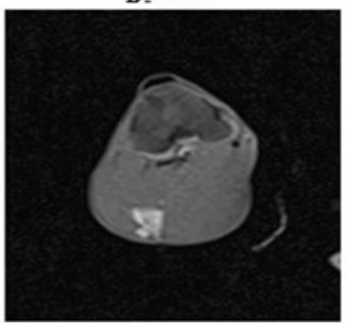

$\mathrm{B}_{4} 3 \mathrm{~h}$
Figure 5 Magnetic resonance images of nest site intravenous injection of dextran-DTPA-Gd in normal rabbits with different time: (B1) 0 min, (B2) $30 \mathrm{~min}$, (B3) $2 \mathrm{~h}$ and (B4) $3 \mathrm{~h}$.

USPIO can be swallowed by phagocytic cells in normal or reactive lymph nodes and accumulated in lymph nodes temporarily, which increases significantly magnetic sensitivity of lymph nodes, and then makes the intensity of magnetic resonance imaging signal increase in lymph nodes. For metastatic lymph nodes, the tumor cells in the lymph nodes can produce the compression, blocking and destruction of lymphatic sinus and hinder the phagocytosis of USPIO by macrophages, which can cause lymph node not to uptake USPIO. Therefore, the benign and malignant lymph nodes can be distinguished. The lymph nodes were imaged with USPIO after intravenous injection of contrast agent (Figure 6). The black arrow was a benign lymph node tumor where the white arrow was a malignant lymph node tumor. The results showed that the signal of benign tumor was weaker and that of the malignant tumor was unchangeable. However, the imaging could be affected by the number and function of macrophages. Because the phagocytic capacity of USPIO relied mainly on the number of macrophages and most passive lymph node containing lots of follicular and few macrophages, the phagocytic ability of lymph nodes was weakened greatly and further false positive results could be obtained. ${ }^{[56]}$ Other researches also presented that the aggregation of USPIO in the lymph nodes was slow, and 24 even $48 \mathrm{~h}$ were needed after the nanoparticles can be imaged.

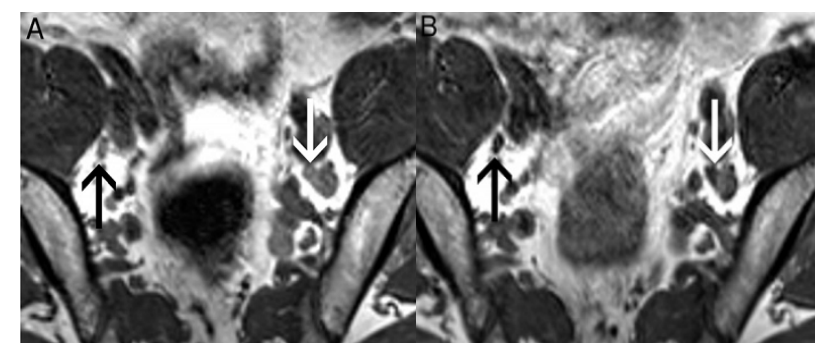

Figure 6 Magnetic resonance images of USPIO uptake in benign and malignant lymph nodes. (A) before and (B) after injection of USPIO.

Hyaluronic acid (HA) is a natural polysaccharide widely distributed in the extracellular matrix. LYVE-1 is a lymph-specific receptor for HA, which can bind both soluble and immobilized HA. Therefore, HA presents a highly promising lymphatic targeting carrier for chemotherapy drugs and diagnostic fluorescence agents. HA-based 
nanomaterials were employed as a safe carrier for the lymphatic targeted delivery of MRI contrast agents. Wu et al. ${ }^{[57]}$ reported a novel MRI contrast agent, hyaluronic acid gadolinium complex (HA-Gd-DTPA) nanospheres. The HA-Gd-DTPA nanospheres have obviously higher relaxation efficiency and a better MRI contrast effect between blood vessel and lymph vessel in rabbit than that of magnevist. Compared to rabbits receiving no injection of contrast agents (Figure 7a), the signal intensity of lymphatic system in rabbits injected with HA-Gd-DTPA nanospheres was obviously enhanced; moreover, the irradiated portions of lymphatic system, such as lymphatic vessel (yellow arrows) and lymph nodes (white arrows), were brighter, and their demarcations become clearer. However, the bright enhancing veins of hind limbs (red arrows) could not be separated from lymphatic vessels in comparision with the rabbits receiving injection of Magnevist after $48 \mathrm{~h}$ (Figure 7b).
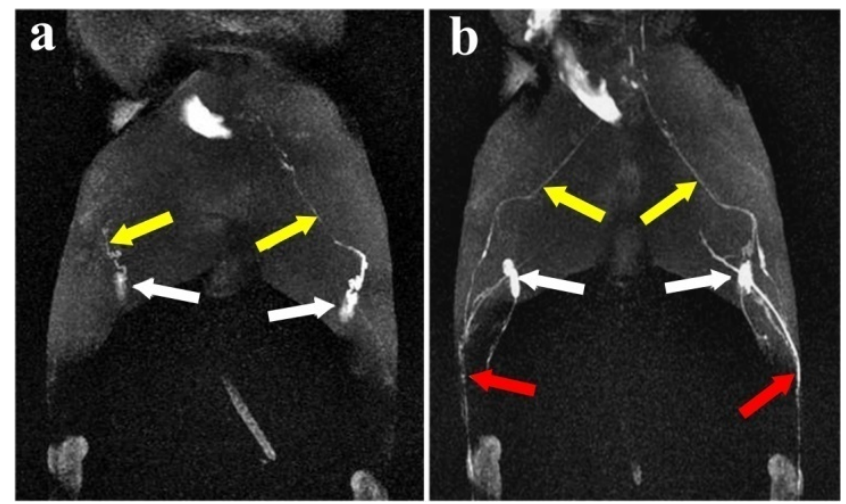

Figure 7 In vivo T1-weighted images of injection with (a) HA-DTPA-Gd nanospheres and (b) magnevist after a $48 \mathrm{~h}$ interval.

Cheng et al ${ }^{[58]}$ prepared a multi-modal lymph-targ- eted contrast agent, hyaluronic acid nanospheres. The ZnO-Gd@HA nanospheres with $19 \%$ of quantum yield and $5.8 \mathrm{mM}^{-1} \cdot \mathrm{s}^{-1}$ of relaxivity could be used for in vivo lymphatic targeting researches. Furthermore, they were highly targeted to lymph system in vitro and in vivo (Figure 8).
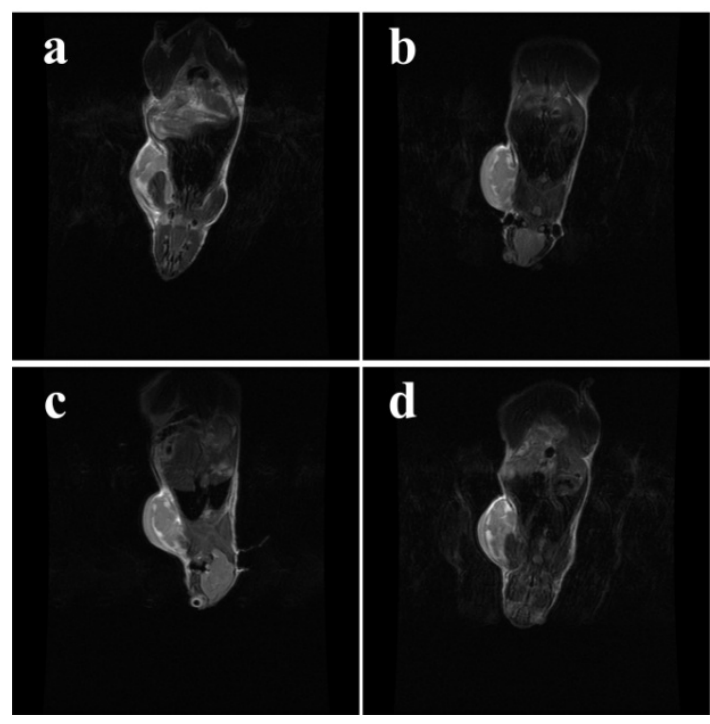

Figure 8 MR images in vivo at different scanning time after post injection. (a) $0 \mathrm{~min}$, (b) $5 \mathrm{~min}$, (c) $15 \mathrm{~min}$, and (d) $25 \mathrm{~min}$.

\section{Conclusion}

The therapeutic efficacy of tumor lymphatic metastasis has aroused widespread concern in the medical field. New technologies will be developed for the diagnosis of tumor metastasis in the future. Satisfactory imaging results were achieved in the targeted contrast agents for lymph nodes presently. However, there are still several drawbacks in the passive targeting mechanism of contrast agents. Therefore, the contrast agents will possess active targeting properties and combine specifically with lymph nodes for improving imaging effects and shortening imaging time. It will realize early diagnosis and early treatment of tumor lymphatic metastasis through structural change of tumor cells in the invasion of lymph nodes. It is believed that the diagnosis of tumor has great value and potential with the rapid progress of imaging and diagnosis, which will bring hope to the treatment of cancer and a broad prospect for development.

\section{Acknowledgement}

The work was supported by the Research Project of the Shanghai Municipal Science and Technology Commission (No. 14411968100), the National Natural Science Foundation of China (Nos. 81271638 and 81371622), and the Shanghai Minhang District Talent Development Special Fund.

\section{References}

[1] DeNardo, D. G.; Johansson, M.; Coussens, L. M. Cancer Metast. Rev. 2008, 27, 11 .

[2] Deryugina, E. I.; Quigley, J. P. Cancer Metast. Rev. 2006, 25, 9.

[3] Ramaswamy, S.; Ross, K. N.; Lander, E. S.; Golub, T. R. Nat. Genet. 2003, 33, 49.

[4] Barbara, M. M.; Ralph, A. R.; Thomas, S. E. Proc. Natl. Acad. Sci. U. S. A. 1992, 89, 11832 .

[5] Petrousjka, M.; Elma, E. M.; Casper, H. J. Surg. Trauma Tumor Growth 1998, 227, 242

[6] Marusyk, A.; Tabassum, D. P.; Altrock, P. M.; Almendro, V.; Michor, F.; Polyak, K. Nature 2014, 514, 54.

[7] Patrick, M.; Alain, P. Nat. Rev. Cancer 2006, 6, 449.

[8] Pouliot, F.; Johnson, M.; Wu, L. Trends Mol. Med. 2009, 15, 254

[9] Smith, A. D.; Shah, S. N.; Rini, B. I.; Lieber, M. L.; Remer, E. M. Am. J. Roentgenol. 2010, 194, 1470.

[10] Yamakado, K.; Nakatsuka, A.; Ohmori, S.; Shiraki, K.; Nakano, T.; Ikoma, J.; Adachi, Y.; Takeda, K. J. Vasc. Interv. Radiol. 2002, 13, 1225

[11] Toloza, E. M.; Harpole, L.; McCrory, D. C. Chest 2003, 123, 137S.

[12] Mukesh, G. H.; Jelle, B.; Peter, F. H. N. Engl. J. Med. 2003, 348, 2491.

[13] Karen, K.; Ying, L.; Marcus, B.; Robert, S.; Warren, R. F. Radiology 2002, 224, 748 .

[14] Ma, L.; Teruya-Feldstein, J.; Weinberg, R. A. Nature 2007, 449, 682.

[15] Skobe, M., Hawighorst, T., Jackson, D. G. Nat. Med. 2001, 7, 192.

[16] Torchilin, V. Adv. Drug Deliv. Rev. 2011, 63,131.

[17] Tan, M. Q.; Burden-Gulley, S. M.; Li, W. Pharm. Res. 2012, 29, 953.

[18] Anderson, C. R.; Hu, X. W.; Zhang, H.; Tlaxca, J. Invest. Radiol. 2011 $46,215$.

[19] Ye, M. Z.; Qian, Y.; Tang, J. B.; Hu, H. J. J. Control. Release 2013, 3 239.

[20] Mattrey, R. F.; Kono, Y.; Baker, K.; Peterson, T. Acad. Radiol. 2002, 9, 231.

[21] Hirakawa, S.; Kodama, S.; Kunstfeld, R.; Kajiya, K.; Brown, L. F.; Detmar, M. J. Exp. Med. 2005, 201, 1089.

[22] Cao, R.; Bjorndahl, M. A.; Religa, P.; Clasper, S.; Garvin, S.; Galter, D.; Meister, B.; Ikomi, F.; Tritsaris, K.; Dissing, S.; Ohhashi, T.; Jackson, D G.; Cao, Y. Cancer Cell 2004, 6, 333.

[23] Katsuta, M.; Miyashita, M.; Makino, H.; Nomura, T. Exp. Mol. Pathol. 2005, 78, 123 .

[24] Datta, J.; Majumder, S.; Kutay, H.; Motiwala, T.; Frankel, W.; Costa, R.; Cha, H. C.; MacDougald, O. A.; Jacob, S. T.; Ghoshal, K. Cancer Res. 2007, 67, 2736

[25] Jain, R. K.; Tong, R. T.; Munn, L. L. Cancer Res. 2007, 67, 2729. 
[26] Jaya K., Vladimir K., Steffen A., Martin H., Debbie W. Cancer Res. 2003, 63, 713.

[27] Ran, S.; Volk, L.; Hall, K. Pathophysiology 2010, 17, 229.

[28] Harrell, J. C.; Dye, W. W.; Allred, D. C. Cancer Res. 2006, 66, 9308.

[29] Chen, Y. B.; Liao, J.; Xie, R. Abdom. Imaging 2011, 36, 102.

[30] Fujiwara, T.; Yasufuku, K.; Nakajima, T. Chest J. 2010, 138, 641.

[31] Bluemel, C.; Schnelzer, A.; Okur, A. Eur. J. Nucl. Med. Mol. Imaging 2013, 40, 1656

[32] Hede, K. J. Natl. Cancer Inst. 2004, 96, 1120.

[33] Fang, J. S.; Gillies, R. D.; Gatenby, R. A. Semin. Cancer. Biol. 2008, 18, 330.

[34] Rahier, J. F.; De Beauce, S.; Dubuquoy, L.; Erdual, E.; Colombel, J. F.; Jouret-Mourin, A.; Geboes, K.; Desreumaux, P. Aliment. Pharmacol. Ther. 2011, 34, 533.

[35] Acs, G.; Paragh, G.; Rakosy, Z. Modern. Pathol. 2012, 25163.

[36] Goldberg, B. B.; Merton, D. A.; Liu, J. B. J. Ultras. Med. 2011, 30, 441.

[37] Schaafsma, B. E.; Mieog, J. S. D.; Hutteman, M. World. J. Surg. Oncol. 2011, 104, 323.

[38] Von, M. Z.; Scholz, A.; Stacker, S. A. Int. J. Oncol. 2005, 27, 669.

[39] Langley, R. R.; Fidler, I. J. Endocr. Rev. 2007, 28, 297.

[40] Jackson, D. G. APMIS 2004, 112, 526.

[41] Witte, M. H.; Jones, K.; Wilting, J. Cancer. Metast. Rev. 2006, 25, 159.

[42] Hirakawa, S.; Brown, L. F.; Kodama, S. Blood 2007, 109, 1010.
[43] Skobe, M.; Hawighorst, T.; Jackson, D.G. Nat. Med. 2001, 7, 192.

[44] Wisner, E.R.; Ferrara, K.W.; Short, R.E. Invest. Radiol. 2003, 38, 358.

[45] Niu, C.; Wang, Z.; Lu, G. Biomaterials 2013, 34, 2307.

[46] Liu, T.; Wu, G. Y.; Cheng, J. J.; Lu, Q.; Yao, Y. J.; Liu, Z. J.; Zhu, D. C.; Zhou, J.; Xu. J. R.; Zhu, J.; He, D. N. Nano Res. 2016, 9, 473.

[47] Harika, L.; Weissleder, R.; Poss, K.; Papisov, M. I. Radiology 1996, 198, 365.

[48] Herborn, C. U.; Vogt, F. M.; J. Magn. Reson. Imaging 2003, 18, 328.

[49] Yan, G. P.; Xu, W.; Yang, L. Pharmaceut Res. 2010, 27, 1884.

[50] Lambregts, D. M.; Beets, G. L.; Maas, M. Ann. Surg. 2011, 253, 539.

[51] Michel, S. C.; Keller, T. M.; Fröhlich, J. M.; Fink, D.; Caduff, R.; Seifert, B.; Marincek, B.; Kubik-Huch, R. A. Radiology 2002, 225, 527.

[52] Triantafyllou, M. S.; Urs, E. B. Eur. J. Cancer 2013.

[53] Saokar, A.; Braschi, M.; Harisinghani, M. Abdom. Imaging 2006, 31, 660.

[54] Azoulay, R.; Olivier, P.; Baud, O.; Verney, C. J. Magn. Reson. Imaging 2008, 28, 1046.

[55] Yan, G. P.; Xu, W.; Yang, L. Pharm. Res. 2010, 27, 1884.

[56] Bellin, M. F.; Beigelman, C. Eur. J. Radiol. 2000, 34, 257.

[57] Wu, G. Y.; Zhang, H. J.; Zhan, Z. F.; Lu, Q.; Cheng, J. J.; Xu, J. R.; Zhu, J. Chin. J. Chem. 2015, 33, 1153 .

[58] Cheng, J. J.; Zhang, H. J.; Zhu J.; Qian L. J.; Lu, Q.; Wu, G. Y.; Xu, J. R.; Zhu, J. Gen. Chem. 2016, 2, 31 . 